\title{
TWO APPROACHES TO MÖBIUS INVERSION
}

\author{
I-CHIAU HUANG
}

(Received 21 April 2011)

\begin{abstract}
The Möbius inversion formula for a locally finite partially ordered set is realized as a Lagrange inversion formula. Schauder bases are introduced to interpret Möbius inversion.
\end{abstract}

2010 Mathematics subject classification: primary 05A19; secondary 06A11, 13D45.

Keywords and phrases: inverse relation, Lagrange inversion, Möbius inversion, Schauder basis.

\section{Introduction}

In combinatorics, there are various inversion formulas including Lagrange inversion, inverse relations and Möbius inversion. Some theorems are in fact also inversions, for instance: MacMahon's master theorem [12]; Saalschütz's theorem [1, p. 9]; (a terminating form of) Dixon's theorem [1, p. 13]; and Jacobi's formula [10]. An inversion formula is regarded as a phenomenon of change of 'coordinate systems'. It is shown that Lagrange inversion [5], MacMahon's master theorem [4, Example 1], Saalschütz's theorem [6, Identity 11] and Dixon's theorem [6, Identity 12] are phenomena of changes of variables; an inverse relation is a phenomenon of a change of Schauder bases [7]; Jacobi's formula is a phenomenon of a change of parameters [9]. The purpose of this article is to show that Möbius inversion for a locally finite partially ordered set is within the same view.

Throughout the article, $\kappa$ is a field and $X$ is a partially ordered set, which is locally finite (that is, there are only finitely many elements between any $x, y \in X$ ). Let $\chi^{n}: X \times X \rightarrow \kappa$ be the function such that $\chi^{n}(x, y)$ is the number of distinct chains $x=x_{0}<x_{1}<\cdots<x_{n}=y$. Since $X$ is locally finite, there is an integer $n$ for each pair $(x, y)$ such that $\chi^{i}(x, y)=0$ for $i>n$, so we can define the Möbius function of $X$ as

$$
\mu:=\chi^{0}-\chi^{1}+\chi^{2}-\chi^{3}+\cdots
$$

For $x \in X$, we call the set $\{y \in X: y \geq x\}$ the principal filter on $X$ generated by $x$. Let $f: X \rightarrow \kappa$ be a function. If all principal filters on $X$ are finite, we can define a

(C) 2011 Australian Mathematical Publishing Association Inc. 0004-9727/2011 \$16.00 
function $g(x):=\sum_{y \geq x} f(y)$; the Möbius inversion formula asserts that

$$
f(x)=\sum_{y \geq x} g(y) \mu(x, y) .
$$

Dually, we call the set $\{y \in X: y \leq x\}$ the principal ideal on $X$ generated by $x$. If all principal ideals on $X$ are finite, we can define a function $g(x):=\sum_{y \leq x} f(y)$; the dual version of the Möbius inversion formula asserts that

$$
f(x)=\sum_{y \leq x} g(y) \mu(y, x) .
$$

Möbius inversion first occurred in number theory. Its significance in combinatorics is shown by Rota [15] by introducing the incidence algebra of a locally finite partially ordered set. In terms of the incidence algebra, the function $\chi^{n}$ is indeed the $n$th power of $\chi^{1}$; the Möbius function $\mu$ is the inverse of the zeta function $\chi^{0}+\chi^{1}$. Observed by Stanley [17], a locally finite partially ordered set can be recovered by its incidence algebra over a field. Incidence algebras are a subject of much research. See the textbook [16]. This article provides alternative views on Möbius inversion by enhancing the method of generating functions with differentials. See [8] for a review on such a method.

Our approach to Möbius inversion as well as to other inversion formulas is supported by convenient operational tools: local cohomology residues are available for changes of variables and certain Schauder bases; for changes of parameters, we can employ logarithmic residues. In Section 2, we briefly recall the notion of local cohomology residues, which is used in this article.

For a fixed $x$, there are only finitely many $y$ involved in the formulas (1.1) and (1.2). Hence we may assume that $X$ is finite for Möbius inversion and its dual version. For the power series over $\kappa$ with the elements of $X$ as variables, we can construct other sets of variables using the order on $X$ (Lemma 3.1). The Möbius function can be computed by residues (equation (3.2) in the proof of Theorem 3.2). As a type of Lagrange inversion, we recover the Möbius inversion formula in Section 3 as the interplay of the representations of a homogeneous polynomial of degree one by these sets of variables. Regarding Lagrange inversion and incidence algebras, another viewpoint is provided by Haiman and Schmitt [2].

Without assuming that $X$ is finite, the set of functions from $X$ to $\kappa$ can be uniquely represented by a Schauder basis (Definition 4.1). For $X$ with finite principal filters or with finite principal ideals, we can define other Schauder bases using the order on $X$ (Propositions 4.2 and 4.3). Section 4 concludes that Möbius inversion is in fact a phenomenon of a change of Schauder bases.

In Section 5, certain computational aspects of our approaches to Möbius functions are presented, including a determinant formula of Lindström and Wilf [11, 18] and another of Redheffer and Wilf [14, 19].

\section{Operational tool}

The concrete features of local cohomology residues have been used in algebraic geometry to realize Grothendieck duality. It is first observed in [4] that these algebraic 
residues are a natural tool for taking coefficients of a power series. With elegant operational rules, local cohomology residues are applied to various combinatorial problems [6]. In this section, we collect basic notions of local cohomology residues used in this article. More details can be found in [4, 6, 8]. See also [3] for a treatment in a more general setting.

A power series ring $R$ over $\kappa$ can be characterized as a complete regular local ring with $\kappa$ as a coefficient field. A regular system of parameters $x_{1}, \ldots, x_{n}$ of $R$ serves as variables. In other words, $R$ can be described as $\kappa\left[\left[x_{1}, \ldots, x_{n}\right]\right]$. The module $\widetilde{\Omega}_{R / \kappa}$ of finite differentials of $R$ over $\kappa$ is free of rank $n$. It comes with a $\kappa$-derivation $d: R \rightarrow \widetilde{\Omega}_{R / \kappa}$, with which $\widetilde{\Omega}_{R / K}=R d x_{1}+\cdots+R d x_{n}$. The exterior power $\wedge^{n} \widetilde{\Omega}_{R / \kappa}$ is free of rank one. Indeed, $\wedge^{n} \widetilde{\Omega}_{R / \kappa}=R d x_{1} \wedge \cdots \wedge d x_{n}$.

The $n$th local cohomology module of $\wedge^{n} \widetilde{\Omega}_{R / \kappa}$ supported at the maximal ideal of $R$ consists of generalized fractions

$$
\left[\begin{array}{c}
\varphi \\
f_{1}, \ldots, f_{n}
\end{array}\right]
$$

where the numerator $\varphi \in \wedge^{n} \widetilde{\Omega}_{R / \kappa}$ and the denominators $f_{1}, \ldots, f_{n}$ form a system of parameters of $R$. Generalized fractions are linear in numerators (linearity law). A change of denominators is compensated by a determinant in the numerator (transformation law). A generalized fraction vanishes if and only if the ideal generated by denominators contains the power series, which represents the numerator in terms of the basis $d x_{1} \wedge \cdots \wedge d x_{n}$ (vanishing law).

Taking residues of a generalized fraction is a $\kappa$-linear map determined by

$$
\operatorname{res}\left[\begin{array}{c}
d x_{1} \wedge \cdots \wedge d x_{n} \\
x_{1}^{i_{1}}, \ldots, x_{n}^{i_{n}}
\end{array}\right]= \begin{cases}1 & \text { if } i_{1}=\cdots=i_{n}=1, \\
0 & \text { otherwise. }\end{cases}
$$

Residue maps are transitive in variables (transitivity law). The most important property of residue maps is that they are invariant under changes of regular systems of parameters (invariance law). We look at an example to see how the invariance law appears explicitly. Consider the power series ring $\kappa[[x]]=\kappa[[y]]$, where $y=$ $x+x^{2}+x^{3}+\cdots$. Leibniz's rule of derivations gives

$$
d y=d x(1-x)^{-1}=(1-x)^{-2} d x .
$$

Hence

$$
\Theta:=\left[\begin{array}{l}
d y \\
y^{n}
\end{array}\right]=\left[\begin{array}{c}
(1-x)^{n-2} d x \\
x^{n}
\end{array}\right]
$$

by the transformation law. For $n=1$, linearity and vanishing laws give

$$
\Theta=\left[\begin{array}{c}
\left(1+x+x^{2}+\cdots\right) d x \\
x
\end{array}\right]=\left[\begin{array}{c}
d x \\
x
\end{array}\right]+\left[\begin{array}{c}
\left(x+x^{2}+\cdots\right) d x \\
x
\end{array}\right]=\left[\begin{array}{c}
d x \\
x
\end{array}\right] ;
$$

for $n \geq 2$, the power series $(1-x)^{n-2}$ becomes a polynomial of degree $n-2$. Thus the residue of the generalized fraction $\Theta$ computed in terms of the variable $y$ is indeed the same as that obtained in terms of $x$. 


\section{Möbius function by residues}

In this section, we work on a power series ring $R=\kappa\left[\left[x_{1}, \ldots, x_{n}\right]\right]$, whose variables $x_{1}, \ldots, x_{n}$ form a partially ordered set $X$. We use the notation

$$
u_{i}:=\sum_{x_{j} \leq x_{i}} x_{j} \quad \text { and } \quad v_{i}:=\sum_{x_{j} \geq x_{i}} x_{j}
$$

for $1 \leq i \leq n$.

Lemma 3.1. The elements $u_{1}, \ldots, u_{n}$ (respectively $v_{1}, \ldots, v_{n}$ ) form a regular system of parameters of $R$. In other words,

$$
\kappa\left[\left[x_{1}, \ldots, x_{n}\right]\right]=\kappa\left[\left[u_{1}, \ldots, u_{n}\right]\right]=\kappa\left[\left[v_{1}, \ldots, v_{n}\right]\right] .
$$

Proof. We prove the lemma for $u_{i}$ and leave the case for $v_{i}$ to the reader. As an application of Nakayama's lemma [13, Theorem 2.3], it suffices to prove that $u_{1}, \ldots, u_{n}$ form a basis of the $\kappa$-vector space generated by $x_{1}, \ldots, x_{n}$. We proceed by induction on the number of variables. The case $n=1$ is trivial. Assume that the assertion holds if the number of variables is less than $n$ and, without loss of generality, assume also that $x_{n}$ is a maximal element. Then $u_{1}, \ldots, u_{n-1}$ form a basis of the vector space generated by $x_{1}, \ldots, x_{n-1}$. The assertion follows since the subspace generated by $u_{1}, \ldots, u_{n}$ is also generated by $u_{1}, \ldots, u_{n-1}, x_{n}$.

The above proof shows that the relation between $u_{1}, \ldots, u_{n}$ (respectively $\left.v_{1}, \ldots, v_{n}\right)$ and $x_{1}, \ldots, x_{n}$ is linear over $\kappa$.

THEOREM 3.2. For $1 \leq j \leq n$,

$$
x_{j}=\sum_{i=1}^{n} \mu\left(x_{i}, x_{j}\right) u_{i}=\sum_{i=1}^{n} \mu\left(x_{j}, x_{i}\right) v_{i} .
$$

Proof. We prove the theorem for $u_{i}$ and leave the case for $v_{i}$ to the reader. Since $x_{j}$ is a $\kappa$-linear combination of $u_{1}, \ldots, u_{n}$, what we need to prove is that

$$
\operatorname{res}\left[\begin{array}{c}
x_{j} d u_{1} \wedge \cdots \wedge d u_{n} \\
u_{1}, \ldots, u_{i-1}, u_{i}^{2}, u_{i+1}, \ldots, u_{n}
\end{array}\right]=\mu\left(x_{i}, x_{j}\right) .
$$

By switching the indices of the sequence $x_{1}, \ldots, x_{n}$, we only need to prove that

$$
\operatorname{res}\left[\begin{array}{c}
x_{n} d u_{1} \wedge \cdots \wedge d u_{n} \\
u_{1}^{2}, u_{2}, \ldots, u_{n}
\end{array}\right]=\mu\left(x_{1}, x_{n}\right),
$$

which clearly holds if $x_{n}$ is a minimal element.

Therefore, we assume that $x_{n}$ is not a minimal element. If $X$ has a minimal element not equal to $x_{1}$, say $x_{2}$, we let $u_{i}^{\prime}=u_{i}-x_{2}$ for $x_{i}>x_{2}$ and let $u_{i}^{\prime}=u_{i}$ otherwise. Then

$$
\left[\begin{array}{c}
x_{n} d u_{1} \wedge \cdots \wedge d u_{n} \\
u_{1}^{2}, u_{2}, \ldots, u_{n}
\end{array}\right]=\left[\begin{array}{c}
x_{n} d u_{1}^{\prime} \wedge d x_{2} \wedge d u_{3}^{\prime} \wedge \cdots \wedge d u_{n}^{\prime} \\
\left(u_{1}^{\prime}\right)^{2}, x_{2}, u_{3}^{\prime}, \ldots, u_{n}^{\prime}
\end{array}\right] .
$$


By the transitivity law of residues, we get

$$
\operatorname{res}\left[\begin{array}{c}
x_{n} d u_{1} \wedge \cdots \wedge d u_{n} \\
u_{1}^{2}, u_{2}, \ldots, u_{n}
\end{array}\right]=\operatorname{res}\left[\begin{array}{c}
x_{n} d u_{1}^{\prime} \wedge d u_{3}^{\prime} \wedge \cdots \wedge d u_{n}^{\prime} \\
\left(u_{1}^{\prime}\right)^{2}, u_{3}^{\prime}, \ldots, u_{n}^{\prime}
\end{array}\right] .
$$

Since $\mu\left(x_{1}, x_{n}\right)$ is invariant if we take away a minimal element not equal to $x_{1}$ from $X$, to prove (3.3), we may assume furthermore that $x_{1}=\inf X$.

Now we prove the theorem by induction on $n$. The case $n=1$ is trivial. Assume the theorem holds for the case that the number of variables is less than $n$. Consider a minimal element of $X \backslash\left\{x_{1}\right\}$, say $x_{2}$. The generalized fraction in (3.3) can be written as

$$
\begin{aligned}
{\left[\begin{array}{c}
x_{n} d u_{1} \wedge \cdots \wedge d u_{n} \\
u_{1}^{2}, u_{2}, \ldots, u_{n}
\end{array}\right]=} & {\left[\begin{array}{c}
x_{n} d x_{1} \wedge\left(d x_{1}+d x_{2}\right) \wedge d u_{3} \wedge \cdots \wedge d u_{n} \\
x_{1}^{2}, x_{1}+x_{2}, u_{3}, \ldots, u_{n}
\end{array}\right] } \\
= & {\left[\begin{array}{c}
x_{n}\left(x_{1}-x_{2}\right) d x_{1} \wedge d x_{2} \wedge d u_{3} \wedge \cdots \wedge d u_{n} \\
x_{1}^{2}, x_{1}^{2}-x_{2}^{2}, u_{3}, \ldots, u_{n}
\end{array}\right] } \\
= & {\left[\begin{array}{c}
x_{n} d x_{1} \wedge d x_{2} \wedge d u_{3} \wedge \cdots \wedge d u_{n} \\
x_{1}^{2}, x_{2}, u_{3}, \ldots, u_{n}
\end{array}\right] } \\
& -\left[\begin{array}{c}
x_{n} d x_{1} \wedge d x_{2} \wedge d u_{3} \wedge \cdots \wedge d u_{n} \\
x_{1}, x_{2}^{2}, u_{3}, \ldots, u_{n}
\end{array}\right] .
\end{aligned}
$$

Let $u_{i}^{\prime}=u_{i}-x_{2}$ for $x_{2}<x_{i}$ and let $u_{i}^{\prime}=u_{i}$ otherwise. Let $u_{i}^{\prime \prime}=u_{i}-x_{1}$. Then

$$
\begin{aligned}
{\left[\begin{array}{c}
x_{n} d u_{1} \wedge \cdots \wedge d u_{n} \\
u_{1}^{2}, u_{2}, \ldots, u_{n}
\end{array}\right]=} & {\left[\begin{array}{c}
x_{n} d x_{1} \wedge d x_{2} \wedge d u_{3}^{\prime} \wedge \cdots \wedge d u_{n}^{\prime} \\
x_{1}^{2}, x_{2}, u_{3}^{\prime}, \ldots, u_{n}^{\prime}
\end{array}\right] } \\
& -\left[\begin{array}{c}
x_{n} d x_{1} \wedge d x_{2} \wedge d u_{3}^{\prime \prime} \wedge \cdots \wedge d u_{n}^{\prime \prime} \\
x_{1}, x_{2}^{2}, u_{3}^{\prime \prime}, \ldots, u_{n}^{\prime \prime}
\end{array}\right]
\end{aligned}
$$

Taking residues, we get

$$
\begin{aligned}
\operatorname{res}\left[\begin{array}{c}
x_{n} d u_{1} \wedge \cdots \wedge d u_{n} \\
u_{1}^{2}, u_{2}, \ldots, u_{n}
\end{array}\right]=\operatorname{res} & {\left[\begin{array}{c}
x_{n} d x_{1} \wedge d u_{3}^{\prime} \wedge \cdots \wedge d u_{n}^{\prime} \\
x_{1}^{2}, u_{3}^{\prime}, \ldots, u_{n}^{\prime}
\end{array}\right] } \\
& -\operatorname{res}\left[\begin{array}{c}
x_{n} d x_{2} \wedge d u_{3}^{\prime \prime} \wedge \cdots \wedge d u_{n}^{\prime \prime} \\
x_{2}^{2}, u_{3}^{\prime \prime}, \ldots, u_{n}^{\prime \prime}
\end{array}\right]
\end{aligned}
$$

Let $\mu^{\prime}$ be the Möbius function of the partially ordered set $X \backslash\left\{x_{2}\right\}$ and let $\mu^{\prime \prime}$ be the Möbius function of the partially ordered set $X \backslash\left\{x_{1}\right\}$. By the induction hypothesis,

$$
\operatorname{res}\left[\begin{array}{c}
x_{n} d u_{1} \wedge \cdots \wedge d u_{n} \\
u_{1}^{2}, u_{2}, \ldots, u_{n}
\end{array}\right]=\mu^{\prime}\left(x_{1}, x_{n}\right)-\mu^{\prime \prime}\left(x_{2}, x_{n}\right)=\mu\left(x_{1}, x_{n}\right) \text {. }
$$

This concludes the proof.

Corollary 3.3. If $x \neq z$, then $\sum_{x \leq y \leq z} \mu(y, z)=\sum_{x \leq y \leq z} \mu(x, y)=0$. 
Proof. We may assume that $X$ consists of elements $x_{1}, \ldots, x_{n}$ with $x=x_{1}=\inf X$ and $z=x_{n}=\sup X$. Then

$$
\begin{aligned}
\sum_{x \leq y \leq z} \mu(y, z) & =\sum_{j=1}^{n} \operatorname{res}\left[\begin{array}{c}
x_{j} d v_{1} \wedge \cdots \wedge d v_{n} \\
v_{1}, \ldots, v_{n-1}, v_{n}^{2}
\end{array}\right] \\
& =\operatorname{res}\left[\begin{array}{c}
v_{1} d v_{1} \wedge \cdots \wedge d v_{n} \\
v_{1}, \ldots, v_{n-1}, v_{n}^{2}
\end{array}\right]=0
\end{aligned}
$$

and

$$
\begin{aligned}
\sum_{x \leq y \leq z} \mu(x, y) & =\sum_{j=1}^{n} \operatorname{res}\left[\begin{array}{c}
x_{j} d u_{1} \wedge \cdots \wedge d u_{n} \\
u_{1}^{2}, u_{2}, \ldots, u_{n}
\end{array}\right] \\
& =\operatorname{res}\left[\begin{array}{c}
u_{n} d u_{1} \wedge \cdots \wedge d u_{n} \\
u_{1}^{2}, u_{2}, \ldots, u_{n}
\end{array}\right]=0 .
\end{aligned}
$$

This concludes the proof.

Consider the power series ring $\kappa\left[\left[x_{1}, \ldots, x_{n}\right]\right]=\kappa\left[\left[y_{1}, \ldots, y_{n}\right]\right]$. Given a power series $f$ represented as

$$
f=\sum a_{i_{1} \cdots i_{n}} x_{1}^{i_{1}} \cdots x_{n}^{i_{n}}
$$

with $a_{i_{1} \cdots i_{n}} \in \kappa$, Lagrange inversion seeks formulas of $b_{i_{1} \cdots i_{n}} \in \kappa$ for a new representation

$$
f=\sum b_{i_{1} \cdots i_{n}} y_{1}^{i_{1}} \cdots y_{n}^{i_{n}}
$$

in terms of $a_{i_{1} \cdots i_{n}}$ and the coefficients $c_{i_{1} \cdots i_{n}}^{(j)}$ in

$$
y_{j}=\sum c_{i_{1} \cdots i_{n}}^{(j)} x_{1}^{i_{1}} \cdots x_{n}^{i_{n}}
$$

With the partial order on the variables $x_{1}, \ldots, x_{n}$, the Möbius inversion formula is a Lagrange inversion formula: in the power series ring $\kappa\left[\left[x_{1}, \ldots, x_{n}\right]\right]=\kappa\left[\left[u_{1}, \ldots, u_{n}\right]\right]$, consider $f:=\sum a_{j} x_{j}$. The elements

$$
b_{i}:=\operatorname{res}\left[\begin{array}{c}
f d u_{1} \wedge \cdots \wedge d u_{n} \\
u_{1}, \ldots, u_{i-1}, u_{i}^{2}, u_{i+1}, \ldots, u_{n}
\end{array}\right]=\sum a_{j} \mu\left(x_{i}, x_{j}\right)
$$

give a new representation $f=\sum b_{i} u_{i}$.

\section{Schauder bases}

The set of functions from $X$ to $\kappa$ can be identified with the set $\bar{I}(X)$ consisting of formal sums $\sum_{x \in X} a_{x} x$ with $a_{x} \in \kappa$. With termwise addition and scalar multiplication, $\bar{I}(X)$ is a vector space over $\kappa$. We remark that $\bar{I}(X)$ can also be identified with the incidence algebra of $X$ modulo its Jacobson radical [17]. Given $f=\sum a_{x} x \in \bar{I}(X)$, the subset

$$
\operatorname{supp} f:=\left\{x \in X: a_{x} \neq 0\right\}
$$

of $X$ is called the support of $f$. 
Definition 4.1. Elements $u_{i} \in \bar{I}(X)$ form a Schauder basis of $\bar{I}(X)$ if the following conditions hold.

- $\quad$ There are only finitely many $u_{i}$ whose support contains any given $x \in X$. (So we can define $\sum a_{i} u_{i}$ for any $a_{i} \in \kappa$.)

- $\quad$ Any element $\bar{I}(X)$ can be written uniquely as $\sum a_{i} u_{i}$ for $a_{i} \in \kappa$.

If $X$ is finite, the notion of Schauder bases agrees with the notion of bases of a vector space. If $X$ is the set of monomials in the power series ring $\kappa[[x]]$ with the order $x^{i}<x^{j} \Leftrightarrow i<j$, then $\bar{I}(X)$ can be identified with $\kappa[[x]]$ as a vector space. In $[7$, Definition 2.1], a slightly different definition of Schauder bases is given for $\kappa[[x]]$. One can show that a Schauder basis of $\bar{I}(X)$ in the sense of this article is countable and can be listed as a Schauder basis $f_{0}, f_{1}, f_{2}, \ldots$ of $\kappa[[x]]$ in the sense of [7].

Proposition 4.2. Assume that all principal filters on $X$ are finite. The elements $u_{y}:=\sum_{x \leq y} x$, where $y \in X$, form a Schauder basis of $\bar{I}(X)$.

Proof. For any $x \in X$, the set consisting of those $y \in X$ such that $x \in \operatorname{supp} u_{y}$ is exactly the principal filter generated by $x$; hence is finite.

We need to represent $\sum_{z} a_{z} z \in \bar{I}(X)$ in terms of $u_{y}$. Recall that Corollary 3.3 asserts that $\sum_{x \leq y \leq z} \mu(y, z)=0$ for $x \neq z$. Together with the fact that $\mu(z, z)=1$, we have $z=\sum_{y \leq z} \mu(y, z) u_{y}$. Therefore,

$$
\sum_{y}\left(\sum_{y \leq z} a_{z} \mu(y, z)\right) u_{y}=\sum_{z} a_{z} z
$$

For the uniqueness of representations, it suffices to show that all $b_{x} \in \kappa$ vanish if $\sum_{x} b_{x} u_{x}=0$. For $y \in X$, the elements $a_{y}:=\sum_{y \leq z} b_{z}$ have to be zero, since $\sum_{y} a_{y} y=$ $\sum_{z} b_{z} u_{z}=0$. On the other hand,

$$
b_{x}=\sum_{x \leq y \leq z} \mu(x, y) b_{z}=\sum_{x \leq y} \mu(x, y) a_{y}=0 .
$$

This concludes the proof.

Similarly we can show the following.

Proposition 4.3. Assume that all principal ideals on $X$ are finite. The elements $v_{y}:=\sum_{x \geq y} x$, where $y \in X$, form a Schauder basis of $\bar{I}(X)$.

Recall that an inverse relation is a pair of identities of the form

$$
\left\{\begin{array}{l}
b_{n}=\sum_{k=0}^{n} c_{n k} a_{k}, \\
a_{n}=\sum_{k=0}^{n} d_{n k} b_{k},
\end{array}\right.
$$


where $a_{i}, b_{i}, c_{j i}, d_{j i} \in \kappa$. It is shown in [7, Theorem 2.1] that an inverse relation with the orthogonal property comes from representations of a power series by two Schauder bases in the sense of [7]. Assume that all principal filters on $X$ are finite. Möbius inversion consist of the information

$$
\left\{\begin{array}{l}
b_{x}=\sum_{y \geq x} \mu(x, y) a_{y}, \\
a_{x}=\sum_{y \geq x} b_{y},
\end{array}\right.
$$

which comes from two representations of an element $\sum a_{x} x=\sum b_{x} u_{x} \in \bar{I}(X)$ by the Schauder bases $\{x\}_{x \in X}$ and $\left\{u_{x}\right\}_{x \in X}$.

\section{Computational aspects}

In this section, we provide three examples to exhibit how differentials and residues naturally appear in Möbius functions and their applications.

In the first example, $X$ is the set of integers with the usual order. We identify an integer $i$ with a variable $x_{i}$. By Theorem 3.2,

$$
\mu(n, n+1)=\operatorname{res}\left[\begin{array}{c}
x_{n+1} d x_{n} \wedge d\left(x_{n}+x_{n+1}\right) \\
x_{n}^{2}, x_{n}+x_{n+1}
\end{array}\right] .
$$

The generalized fraction in the above formula can be simplified as

$$
\begin{aligned}
{\left[\begin{array}{c}
x_{n+1} d x_{n} \wedge d\left(x_{n}+x_{n+1}\right) \\
x_{n}^{2}, x_{n}+x_{n+1}
\end{array}\right] } & =\left[\begin{array}{c}
x_{n+1}\left(x_{n}-x_{n+1}\right) d x_{n} \wedge d x_{n+1} \\
x_{n}^{2}, x_{n}^{2}-x_{n+1}^{2}
\end{array}\right] \\
& =\left[\begin{array}{c}
x_{n+1}\left(x_{n}-x_{n+1}\right) d x_{n} \wedge d x_{n+1} \\
x_{n}^{2},-x_{n+1}^{2}
\end{array}\right] \\
& =\left[\begin{array}{c}
d x_{n} \wedge d x_{n+1} \\
x_{n},-x_{n+1}
\end{array}\right] .
\end{aligned}
$$

By taking the residue,

$$
\mu(n, n+1)=\operatorname{res}\left[\begin{array}{c}
d x_{n} \wedge d x_{n+1} \\
x_{n},-x_{n+1}
\end{array}\right]=-1 .
$$

For $m>n+1$,

$$
\begin{aligned}
\mu(n, m) & =\operatorname{res}\left[\begin{array}{c}
x_{m} d x_{n} \wedge d\left(x_{n}+x_{n+1}\right) \wedge \cdots \wedge d\left(x_{n}+\cdots+x_{m}\right) \\
x_{n}^{2}, x_{n}+x_{n+1}, \ldots, x_{n}+x_{n+1}+\cdots+x_{m}
\end{array}\right] \\
& =\operatorname{res}\left[\begin{array}{c}
x_{m} d x_{n} \wedge d\left(x_{n}+x_{n+1}\right) \wedge \cdots \wedge d\left(x_{n}+\cdots+x_{m}\right) \\
x_{n}^{2}, x_{n}+x_{n+1}, \ldots, x_{n}+x_{n+1}+\cdots+x_{m-1}, x_{m}
\end{array}\right]=0 .
\end{aligned}
$$

For the rest of this section, we consider a partially ordered finite set $X=\left\{x_{1}, \ldots, x_{n}\right\}$ and use the notation of (3.1) in Section 2. 
In the second example, we revisit a determinant formula obtained independently by Lindström [11] and Wilf [18]. For a sequence $a_{1}, \ldots, a_{n} \in \kappa$, the differential

$$
\omega_{i}:=d\left(\sum_{x_{\ell} \geq x_{i}} a_{\ell} u_{\ell}\right)
$$

can be written as

$$
\omega_{i}=\sum_{j=1}^{n} b_{i j} d x_{j}
$$

for $b_{i j} \in \kappa$. Indeed, $b_{i j}=\sum_{x_{\ell} \geq x_{i}, x_{j}} a_{\ell}$. For a maximal element $x_{m}$ of $X$,

$$
\omega_{1} \wedge \cdots \wedge \omega_{n}=\omega_{1}^{\prime} \wedge \cdots \wedge \omega_{m-1}^{\prime} \wedge\left(a_{m} d x_{m}\right) \wedge \omega_{m-1}^{\prime} \wedge \cdots \wedge \omega_{n}^{\prime},
$$

where $\omega_{i}^{\prime}:=d\left(\sum_{x_{i} \leq x_{\ell} \neq x_{m}} a_{\ell} u_{\ell}\right)$. By induction on $n$,

$$
\omega_{1} \wedge \cdots \wedge \omega_{n}=\left(a_{1} d x_{1}\right) \wedge \cdots \wedge\left(a_{n} d x_{n}\right) .
$$

Hence

$$
\operatorname{det}\left(b_{i j}\right)=\operatorname{res}\left[\begin{array}{c}
\omega_{1} \wedge \cdots \wedge \omega_{n} \\
x_{1}, \ldots, x_{n}
\end{array}\right]=a_{1} \cdots a_{n} .
$$

Let $b_{i}:=b_{i i}$. In terms of the Möbius function, the above determinant formula can also be written as

$$
\operatorname{det}\left(b_{i j}\right)=\prod_{i=1}^{n} \sum_{j=1}^{n} \mu\left(x_{i}, x_{j}\right) b_{j} .
$$

For our third example, we recall $u_{i}=\sum_{x_{j} \leq x_{i}} x_{j}$ and $x_{j}=\sum_{i=1}^{n} \mu\left(x_{i}, x_{j}\right) u_{i} . \quad$ The Möbius function also appears in the identity

$$
d x_{j} \wedge d u_{2} \wedge \cdots \wedge d u_{n}=\mu\left(x_{1}, x_{j}\right) d u_{1} \wedge d u_{2} \wedge \cdots \wedge d u_{n}
$$

of exterior products of differentials. Let $R$ be the matrix whose $(i, j)$ entry is 1 if $x_{i} \leq x_{j}$ or $j=1$, and otherwise is 0 . The determinant considered by Redheffer [14] and Wilf [19] can be written as

$$
\operatorname{det} R=\operatorname{res}\left[\begin{array}{c}
d\left(x_{1}+\cdots+x_{n}\right) \wedge d u_{2} \wedge \cdots \wedge d u_{n} \\
x_{1}, \ldots, x_{n}
\end{array}\right] .
$$

To compute the residue, we use the relation

$$
\left(\begin{array}{c}
u_{1} \\
\vdots \\
u_{n}
\end{array}\right)=\left(\begin{array}{ccc}
\left(\chi^{0}+\chi^{1}\right)\left(x_{1}, x_{1}\right) & \cdots & \left(\chi^{0}+\chi^{1}\right)\left(x_{n}, x_{1}\right) \\
\vdots & \ddots & \vdots \\
\left(\chi^{0}+\chi^{1}\right)\left(x_{1}, x_{n}\right) & \cdots & \left(\chi^{0}+\chi^{1}\right)\left(x_{n}, x_{n}\right)
\end{array}\right)\left(\begin{array}{c}
x_{1} \\
\vdots \\
x_{n}
\end{array}\right)
$$

of matrices to change the denominators of the generalized fraction in (5.1) from $x_{1}, \ldots, x_{n}$ to $u_{1}, \ldots, u_{n}$. Note that $\operatorname{det}\left(\left(\chi^{0}+\chi^{1}\right)\left(x_{i}, x_{j}\right)\right)$ is invariant under any 
permutation of the indices of the sequence $x_{1}, \ldots, x_{n}$. Since there is a permutation of the indices of $x_{1}, \ldots, x_{n}$ such that the matrix $\left(\left(\chi^{0}+\chi^{1}\right)\left(x_{i}, x_{j}\right)\right)$ is upper triangular with ones on the diagonal, we have $\operatorname{det}\left(\left(\chi^{0}+\chi^{1}\right)\left(x_{i}, x_{j}\right)\right)=1$. By the transformation law,

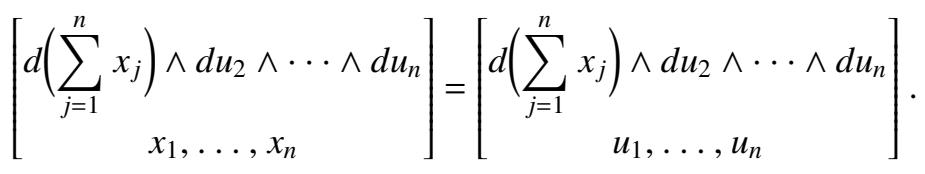

We recover the determinant formula of Redheffer [14] as well as its generalization by Wilf [19] as follows:

$$
\begin{aligned}
& \operatorname{det} R=\operatorname{res}\left[\begin{array}{c}
d\left(\sum_{j=1}^{n} x_{j}\right) \wedge d u_{2} \wedge \cdots \wedge d u_{n} \\
u_{1}, \ldots, u_{n}
\end{array}\right] \\
& =\sum_{j=1}^{n} \operatorname{res}\left[\begin{array}{c}
d x_{j} \wedge d u_{2} \wedge \cdots \wedge d u_{n} \\
u_{1}, \ldots, u_{n}
\end{array}\right]=\sum_{j=1}^{n} \mu\left(x_{1}, x_{j}\right) .
\end{aligned}
$$

We remark that the assumption in [19] that $x_{1}$ is the smallest element of $X$ is irrelevant in the above proof.

\section{References}

[1] W. N. Bailey, Generalized Hypergeometric Series, Cambridge Tracts in Mathematics and Mathematical Physics, 32 (Stechert-Hafner, New York, 1964).

[2] M. Haiman and W. Schmitt, 'Incidence algebra antipodes and Lagrange inversion in one and several variables', J. Combin. Theory Ser. A 50(2) (1989), 172-185.

[3] I-C. Huang, 'Pseudofunctors on modules with zero dimensional support', Mem. Amer. Math. Soc. 114(548) (1995), xii+53.

[4] I-C. Huang, 'Applications of residues to combinatorial identities', Proc. Amer. Math. Soc. 125(4) (1997), 1011-1017.

[5] I-C. Huang, 'Reversion of power series by residues', Comm. Algebra 26(3) (1998), 803-812.

[6] I-C. Huang, 'Residue methods in combinatorial analysis', in: Local Cohomology and its Applications, Lecture Notes in Pure and Applied Mathematics, 226 (Marcel Dekker, New York, NY, 2001), pp. 255-342.

[7] I-C. Huang, 'Inverse relations and Schauder bases', J. Combin. Theory Ser. A 97(2) (2002), 203-224.

[8] I-C. Huang, 'Method of generating differentials', in: Advances in Combinatorial Mathematics: Proceedings of the Waterloo Workshop in Computer Algebra 2008 (Springer, Berlin, 2009), pp. $125-152$.

[9] I-C. Huang, 'Changes of parameters for generalized power series', Comm. Algebra 38 (2010), 2480-2498.

[10] C. G. I. Jacobi, 'De resolutione aequationum per series infinitas', J. reine angew. Math. 6 (1830), 257-286.

[11] B. Lindström, 'Determinants on semilattices', Proc. Amer. Math. Soc. 20 (1969), 207-208.

[12] P. A. MacMahon, Combinatory Analysis (Chelsea, New York, 1960), two volumes (bound as one).

[13] H. Matsumura, Commutative Ring Theory (Cambridge University Press, Cambridge, 1986). 
[14] R. Redheffer, 'Eine explizit lösbare Optimierungsaufgabe', in: Numerische Methoden bei Optimierungsaufgaben, Band 3 (Tagung, Math. Forschungsinst., Oberwolfach, 1976), International Series of Numerical Mathematics, 36 (Birkhäuser, Basel, 1977), pp. 213-216.

[15] G.-C. Rota, 'On the foundations of combinatorial theory. I. Theory of Möbius functions', Z. Wahrscheinlichkeitstheor. Verw. Geb. 2 (1964), 340-368.

[16] E. Spiegel and C. J. O’Donnell, Incidence Algebras, Monographs and Textbooks in Pure and Applied Mathematics, 206 (Marcel Dekker, New York, NY, 1997).

[17] R. P. Stanley, 'Structure of incidence algebras and their automorphism groups', Bull. Amer. Math. Soc. 76 (1970), 1236-1239.

[18] H. S. Wilf, 'Hadamard determinants, Möbius functions, and the chromatic number of a graph', Bull. Amer. Math. Soc. 74 (1968), 960-964.

[19] H. S. Wilf, 'The Redheffer matrix of a partially ordered set', Electron. J. Combin. 11(2) (2004/06), Research Paper 10, 5 pp (electronic).

I-CHIAU HUANG, Institute of Mathematics, Academia Sinica, 6F, Astronomy-Mathematics Building, No. 1, Sec. 4, Roosevelt Road, Taipei 10617, Taiwan, ROC

e-mail: ichuang@math.sinica.edu.tw 